\title{
ON THE CLASSIFICATION OF THE REAL VECTOR SUBSPACES OF A QUATERNIONIC VECTOR SPACE
}

\author{
RADU PANTILIE
}

\begin{abstract}
We prove the classification of the real vector subspaces of a quaternionic vector space by using a covariant functor which, to any pair formed of a quaternionic vector space and a real subspace, associates a coherent sheaf over the sphere.
\end{abstract}

\section{INTRODUCTION}

Let $X_{E}$ be the space of (real) vector subspaces of a vector space $E$. Then $X_{E}$ is a disjoint union of Grassmannians and $\operatorname{GL}(E)$ acts transitively on each of its components.

If $E$ is endowed with a linear geometric structure, corresponding to the Lie subgroup $G \subseteq \operatorname{GL}(E)$, then it is natural to ask whether or not the action induced by $G$ on $X_{E}$ is still transitive on each component and, if not, to find explicit representatives for each orbit.

For example, if $E$ is an Euclidean vector space and, accordingly, $G$ is the orthogonal group then the orthonormalization process shows that $G$ acts transitively on each component of $X_{E}$.

Suppose, instead, that $E$ is endowed with a linear complex structure $J$; equivalently, $E=\mathbb{C}^{k}$ and $G=\operatorname{GL}(k, \mathbb{C})$. Then for any vector subspace $U$ of $E$ we have a decomposition $U=F \times V$, where $F$ is a complex vector subspace of $E$ and $V$ is totally real (that is, $V \cap J V=0$ ); obviously, the filtration $0 \subseteq F \subseteq U$ is canonical. Consequently, the subspaces $\mathbb{C}^{m} \times \mathbb{R}^{l}$, where $2 m+l \leq 2 k$, are representatives for each of the orbits of $\mathrm{GL}(k, \mathbb{C})$ on $X_{\mathbb{C}^{k}}$.

The corresponding decomposition for the real subspaces of a hypercomplex vector space - that is, $E=\mathbb{H}^{k}$ and $G=\mathrm{GL}(k, \mathbb{H})$ - was obtained in [2] .

By using a different method, we obtain the decomposition and the canonical filtration for the real subspaces of a quaternionic vector space; that is, $E=\mathbb{H}^{k}$ and $G=\operatorname{Sp}(1) \cdot \mathrm{GL}(k, \mathbb{H})$. This involves a covariant functor from the category of pairs $(U, E)$, where $E$ is a quaternionic vector space and $U \subseteq E$ is a real vector

2010 Mathematics Subject Classification. 18F20, 53C26, 53C28, 54B40. 
subspace (with the obvious morphisms induced by the linear quaternionic maps), to the category of coherent sheaves on the Riemann sphere. We mention that a similar functor appeared in [7] (see [8] ).

I am grateful to Stefano Marchiafava for useful discussions and comments, and to Anton Galaev for informing me about [2] .

\section{Complex And (CO-)CR vector spaces}

A linear complex structure on a (real) vector space $U$ is a linear map $J: U \rightarrow U$ such that $J^{2}=-\mathrm{Id}_{U}$. Then, on associating to any linear complex structure the $-\mathrm{i}$ eigenspace of its complexification, we obtain a (bijective) correspondence between the space of linear complex structures on $U$ and the space of complex vector subspaces $C$ of $U^{\mathbb{C}}$ such that $C \oplus \bar{C}=U^{\mathbb{C}}$.

This suggests to consider the following two less restrictive conditions for a complex vector subspace $C$ of $U^{\mathbb{C}}$ :

1) $C \cap \bar{C}=0$,

2) $C+\bar{C}=U^{\mathbb{C}}$.

Furthermore, conditions (1) and (2) are dual to each other. That is, $C \subseteq U^{\mathbb{C}}$ satisfies condition (1) if and only if Ann $C \subseteq\left(U^{\mathbb{C}}\right)^{*}$ satisfies (2), where Ann $C=$ $\left\{\alpha \in\left(U^{\mathbb{C}}\right)^{*}|\alpha|_{C}=0\right\}$ is the annihilator of $C$.

Now, it is a standard fact that if $C \subseteq U^{\mathbb{C}}$ satisfies (1) then it is called a linear $C R$ structure on $U$.

Therefore a complex vector subspace $C$ of $U^{\mathbb{C}}$ satisfying $C+\bar{C}=U^{\mathbb{C}}$ is called a linear co-CR structure on $U$ [6] .

Thus, a complex vector subspace of $U^{\mathbb{C}}$ is a linear co-CR structure on $U$ if and only if its annihilator is a linear CR structure on $U^{*}$.

A vector space endowed with a linear (co-)CR structure is a (co-)CR vector space.

If $U$ is a vector subspace of a vector space $E$, endowed with a linear complex structure $J$, then $C=U^{\mathbb{C}} \cap E^{J}$ is a linear CR structure on $U$, where $E^{J}$ is the -i eigenspace of $J$. Moreover, if we further assume $U+J U=E$ then $(E, J)$ is, up to complex linear isomorphisms, the unique complex vector space, containing $U$, such that $C=U^{\mathbb{C}} \cap E^{J}$.

Thus, we have the following fact.

Proposition 1.1 (see [6]). Any CR vector space corresponds to a pair $(U, E)$, where $(E, J)$ is a complex vector space and $U$ is a vector subspace of $E$ such that $U+J U=E$.

We, also, have the following dual fact. 
Proposition 1.2 ([6] ). Any co-CR vector space corresponds to a and pair $(V, E)$, where $(E, J)$ is a complex vector space and $V$ is a vector subspace of $E$ such that $V \cap J V=0$.

Proof. Let $(E, J)$ be a complex vector space and let $V \subseteq E$ be totally real; that is, $V \cap J V=0$. Let $U=E / V$ and let $\pi: E \rightarrow U$ be the projection. Then $\pi\left(E^{J}\right)$ is a linear co-CR structure on $U$ and the proof follows quickly.

Let $(E, J)$ be a complex vector space and let $U$ be a vector subspace of $E$. Then, obviously, $F=U \cap J U$ is invariant under $J$ and therefore $\left(F,\left.J\right|_{F}\right)$ is a complex vector subspace of $(E, J)$. Moreover, $\left(F,\left.J\right|_{F}\right)$ is the biggest complex vector subspace of $(E, J)$ contained by $U$. Consequently, if $V$ is a complement of $F$ in $U$ then $V$ is totally real in $E$.

Thus, we have a decomposition $U=F \oplus V$; moreover, the filtration $0 \subseteq F \subseteq U$ is canonical.

As already suggested, it is useful to consider pairs $(U, E)$, with $E$ a complex vector space and $U$ a vector subspace of $E$. A morphism $t:(U, E) \rightarrow\left(U^{\prime}, E^{\prime}\right)$, between two such pairs, is a complex linear map $t: E \rightarrow E^{\prime}$ such that $t(U) \subseteq U^{\prime}$. Also, there is an obvious notion of product: $(U, E) \times\left(U^{\prime}, E^{\prime}\right)=\left(U \times U^{\prime}, E \times E^{\prime}\right)$.

Proposition 1.3 (see [2] ). Any pair formed of a complex vector space and a real vector subspace admits a decomposition, unique up to the order of factors, as a (finite) product in which each factor is either $(\mathbb{C}, \mathbb{C}),(\mathbb{R}, \mathbb{C})$, or $(0, \mathbb{C})$.

Proof. Let $(E, J)$ be a complex vector space and let $U$ be a vector subspace of $E$. We have seen that $U=F \times V$, where $F=U \cap J U$ and $V$ is a complement of $F$ in $U$. From the fact that $V \cap J V=0$ it follows that $F \cap(V+J V)=0$.

Let $E^{\prime} \subseteq E$ be a complex vector subspace complementary to $F \oplus(V+J V)$. We, obviously, have that $(U, E)$ is isomorphic to $(F, F) \times(V, V+J V) \times\left(0, E^{\prime}\right)$.

To complete the proof, just note that $(F, F),(V, V+J V)$, and $\left(0, E^{\prime}\right)$ decompose as products in which each factor is of the form $(\mathbb{C}, \mathbb{C}),(\mathbb{R}, \mathbb{C})$, and $(0, \mathbb{C})$, respectively.

If we apply Proposition 1.3 to the pair corresponding to a (co-)CR vector space then we obtain the following facts, dual to each other:

1) The pair corresponding to a $\mathrm{CR}$ vector space admits a decomposition, unique up to the order of factors, as a product in which each factor is either $(\mathbb{C}, \mathbb{C})$ or $(\mathbb{R}, \mathbb{C})$;

2) The pair corresponding to a co-CR vector space admits a decomposition, unique up to the order of factors, as a product in which each factor is either 
$(\mathbb{R}, \mathbb{C})$ or $(0, \mathbb{C})$.

Thus, we have the following result.

Corollary 1.4. Any pair formed of a complex vector space and a real vector subspace admits a decomposition as a product of the pair corresponding to a $C R$ vector space and the pair corresponding to a co-CR vector space.

\section{QUATERNIONIC VECTOR SPACES}

The automorphism group of the (unital) associative algebra of quaternions is $\mathrm{SO}(3, \mathbb{R})$, acting trivially on $\mathbb{R}$ and canonically on $\operatorname{Im} \mathbb{H}\left(=\mathbb{R}^{3}\right)$. Thus, if $E$ is a vector space then there exists a natural action of $\mathrm{SO}(3, \mathbb{R})$ on the space of morphisms of associative algebras from $\mathbb{H}$ to $\operatorname{End}(E)$; that is, on the space of linear hypercomplex structures on $E$. The (nonempty) orbits of this action are the linear quaternionic structures on $E$.

A quaternionic (hypercomplex) vector space is a vector space endowed with a linear quaternionic (hypercomplex) structure (see [1] , [4] ).

Let $E$ be a quaternionic vector space and let $\rho: \mathbb{H} \rightarrow \operatorname{End}(E)$ be a representative of its linear quaternionic structure. Then, obviously, the space $Z=\rho\left(S^{2}\right)$ of admissible linear complex structures on $E$ depends only of the linear quaternionic structure of $E$. We denote by $E^{J}$ the - i eigenspace of $J \in Z$.

The linear quaternionic structure on $E$ corresponds to a linear quaternionic structure on its dual $E^{*}$ given by the morphism of associative algebras from $\mathbb{H}$ to $\operatorname{End}\left(E^{*}\right)$, which maps any $q \in \mathbb{H}$ to the transpose of $\rho(\bar{q})$. Thus, any admissible linear complex structure $J$ on $E$ corresponds to the admissible linear complex structure $J^{*}$ which is the opposite of the transpose of $J$; note that, $\left(E^{*}\right)^{J^{*}}$ is the annihilator of $E^{J}$.

Let $E$ and $E^{\prime}$ be quaternionic vector spaces and let $Z$ and $Z^{\prime}$ be the corresponding spaces of admissible linear complex structures, respectively. A linear quaternionic map from $E$ to $E^{\prime}$ is a linear map $t: E \rightarrow E^{\prime}$ such that, for some function $T: Z \rightarrow Z^{\prime}$, we have $t \circ J=T(J) \circ t$, for any $J \in Z$; consequently, if $t \neq 0$ then $T$ is unique and an orientation preserving isometry (see [4] ).

The (left) $\mathbb{H}$-module structure on $\mathbb{H}^{k}$ determines a linear quaternionic structure on it. Moreover, for any quaternionic vector space $E$, with $\operatorname{dim} E=4 k$, there exists a linear quaternionic isomorphism from $E$ onto $\mathbb{H}^{k}$. The group of linear quaternionic automorphisms of $\mathbb{H}^{k}$ is $\operatorname{Sp}(1) \cdot \operatorname{GL}(k, \mathbb{H})$, acting on $\mathbb{H}^{k}$ by $( \pm(a, A), q) \mapsto a q A^{-1}$, for any $\pm(a, A) \in \mathrm{Sp}(1) \cdot \mathrm{GL}(k, \mathbb{H})$ and $q \in \mathbb{H}^{k}$ (see [4] ).

We end this section by showing how to define the product of two quaternionic vector spaces $E$ and $E^{\prime}$. Let $T: Z \rightarrow Z^{\prime}$ be an orientation preserving isometry 
between the spaces of admissible linear complex structures on $E$ and $E^{\prime}$.

If $\rho: \mathbb{H} \rightarrow \operatorname{End}(E)$ represents the linear quaternionic structure of $E$ then $T$ is the restriction of a unique linear map $\widetilde{T}: \rho(\mathbb{H}) \rightarrow \operatorname{End}\left(E^{\prime}\right)$ such that $\widetilde{T} \circ \rho$ determines the linear quaternionic structure on $E^{\prime}$.

Then $q \mapsto(\rho(q), \widetilde{T}(\rho(q))),(q \in \mathbb{H})$, defines the product linear quaternionic structure on $E \times E^{\prime}$ (with respect to $T$ ).

Note that, although the product of two quaternionic vector spaces is welldefined (that is, it doesn't depend on the particular isometry $T$ ), it doesn't make the category of quaternionic vector spaces Abelian. Nevertheless, it is obvious that the category of hypercomplex vector spaces is Abelian.

\section{Pairs Formed of a QUATERnionic VeCtor SPACE AND A REAL VECTOR SUBSPACE}

The category of quaternionic vector spaces is a full subcategory of the category whose objects are pairs $(U, E)$, where $E$ is a quaternionic vector space and $U \subseteq E$ is a real vector subspace. The morphisms between two such pairs $(U, E)$ and $\left(U^{\prime}, E^{\prime}\right)$ are the linear quaternionic maps $t: E \rightarrow E^{\prime}$ such that $t(U) \subseteq U^{\prime}$ (see [2]).

If $U$ is a real vector subspace of a quaternionic vector space $E$ we call (Ann $U, E^{*}$ ) the dual of $(U, E)$.

We shall see that there are three basic subcategories of the category of pairs formed of a quaternionic vector space and a real vector subspace, two of which are related to the Twistor Theory (see [6] ).

Definition 3.1. Let $E$ be a quaternionic vector space and let $Z$ be its space of admissible linear complex structures.

If $\iota: U \rightarrow E$ is an injective linear map then $(E, \iota)$ is a linear $C R$ quaternionic structure on $U$ if $\operatorname{im} \iota+J(\operatorname{im} \iota)=E$, for any $J \in Z$.

A CR quaternionic vector space is a vector space endowed with a linear CR quaternionic structure.

By duality, we obtain the notion of co-CR quaternionic vector space.

To any co-CR quaternionic vector space $(U, E, \rho)$ we associate the pair $(\operatorname{ker} \rho, E)$. Thus, the category of co-CR quaternionic vector spaces is a full subcategory of the category of pairs formed of a quaternionic vector space and a real vector subspace; by duality, the latter, also, includes the category of CR quaternionic vector spaces.

See [6] for further information on (co-)CR quaternionic vector spaces. 
Remark 3.2. 1) Let $U$ be a real vector subspace of a quaternionic vector space $E$. Then $(U, E)$ is given by a $\mathrm{CR}$ quaternionic vector space if and only if its dual is given by a co-CR quaternionic vector space.

2) Any quaternionic vector space $E$ is both $\mathrm{CR}$ and co-CR quaternionic. When we consider $E$ a $\mathrm{CR}$ quaternionic vector space the associated pair is $(E, E)$, whilst when we consider $E$ a co-CR quaternionic vector space the associated pair is $(0, E)$.

We shall construct a covariant functor from the category of pairs, formed of a quaternionic vector space and a real vector subspace, to the category of coherent analytic sheaves, over the sphere, endowed with a conjugation covering the antipodal map (see [3] for the basic properties of coherent analytic sheaves and [7] for coherent analytic sheaves, over the sphere, endowed with a conjugation covering the antipodal map, briefly called ' $\sigma$-sheaves').

For this, firstly, note that if $E$ is a quaternionic vector space, with $Z\left(=S^{2}\right)$ the space of admissible linear complex structures, then $E^{0,1}=\bigcup_{J \in Z}\{J\} \times E^{J}$ is a holomorphic vector subbundle of $Z \times E^{\mathbb{C}}$. Now, if $U \subseteq E$ is a real vector subspace then the projection $E \rightarrow E / U$ induces, by restriction, a morphism of holomorphic vector bundles $E^{0,1} \rightarrow Z \times(E / U)^{\mathbb{C}}$. Let $\mathcal{U}_{-}$and $\mathcal{U}_{+}$be the kernel and cokernel, respectively, of this morphism of holomorphic vector bundles.

Definition 3.3. We call $\mathcal{U}=\mathcal{U}_{-} \oplus \mathcal{U}_{+}$the (coherent analytic) sheaf of $(U, E)$.

The proof of the following proposition is straightforward.

Proposition 3.4. The association $(U, E) \mapsto \mathcal{U}$ defines a covariant functor $\mathscr{F}$ from the category of pairs, formed of a quaternionic vector space $E$ and a real vector subspace $U \subseteq E$, to the category of coherent sheaves, on the sphere, endowed with a conjugation covering the antipodal map. Furthermore, $\mathscr{F}$ has the following properties:

(i) For any morphism $t:(U, E) \rightarrow\left(U^{\prime}, E^{\prime}\right)$, we have that $\mathscr{F}(t)$ maps $\mathscr{F}(U, E)_{ \pm}$ to $\mathscr{F}\left(U^{\prime}, E^{\prime}\right)_{ \pm}$.

(ii) If $(U, E)$ is given by a (co-)CR quaternionic vector space then $\mathscr{F}(U, E)$ is its holomorphic vector bundle.

With the same notations as in Proposition 3.4, if $\mathcal{U}=\mathcal{U}_{+}$then $E / U$ is the space of (global) sections of $\mathcal{U}$ intertwining the antipodal map and the conjugation.

Here are the basic examples of pairs whose sheaves are torsion free (cf. [2] , 7] ; see, also, [8], [6] ). 
Example 3.5. Let $q_{1}, \ldots, q_{k+1} \in S^{2},(k \geq 1)$, be such that $q_{i} \neq \pm q_{j}$, if $i \neq j$. For $j=1, \ldots, k$ let $e_{j}=(\underbrace{0, \ldots, 0}_{j-1}, q_{j}, q_{j+1}, \underbrace{0, \ldots, 0}_{k-j})$. Denote $U_{0}=\mathbb{R}$ and, for $k \geq 1$, let $U_{k}=\mathbb{R}^{k+1}+\mathbb{R} e_{1}+\ldots+\mathbb{R} e_{k}$.

Then the sheaf of $\left(U_{k}, \mathbb{H}^{k+1}\right)$ is $\mathcal{O}(2 k+2)$, for any $k \in \mathbb{N}$. Note that, the projection $\mathbb{H}^{k+1} \rightarrow \mathbb{H}^{k+1} / U_{k}$ defines a co-CR quaternionic vector space and the sheaf of the dual of $\left(U_{k}, \mathbb{H}^{k+1}\right)$ is $\mathcal{O}(-2 k-2)$, for any $k \in \mathbb{N}$.

Example 3.6. Let $V_{0}=\{0\}$ and, for $k \geq 1$, let $V_{k}$ be the vector subspace of $\mathbb{H}^{2 k+1}$ formed of all vectors of the form

$$
\left(z_{1}, \overline{z_{1}}+z_{2} \mathrm{j}, z_{3}-\overline{z_{2}} \mathrm{j}, \ldots, \overline{z_{2 k-1}}+z_{2 k} \mathrm{j},-\overline{z_{2 k}} \mathrm{j}\right),
$$

where $z_{1}, \ldots, z_{2 k}$ are complex numbers.

Then the sheaf of $\left(V_{k}, \mathbb{H}^{2 k+1}\right)$ is $2 \mathcal{O}(2 k+1)$, for any $k \in \mathbb{N}$. Note that, the projection $\mathbb{H}^{2 k+1} \rightarrow \mathbb{H}^{2 k+1} / V_{k}$ defines a co-CR quaternionic vector space and the sheaf of the dual of $\left(V_{k}, \mathbb{H}^{2 k+1}\right)$ is $2 \mathcal{O}(-2 k-1)$, for any $k \in \mathbb{N}$.

The next class of pairs is taken from [2] .

Example 3.7. For $k \geq 1$ and $q \in S^{2}$, let $W_{k, q}$ be the real vector subspace of $\mathbb{H}^{k}$ formed of all vectors of the form

$$
\left(a_{1}+b_{1} q+b_{2} \mathrm{i}, a_{2}+b_{2} q+b_{3} \mathrm{i}, \ldots, a_{k-1}+b_{k-1} q+b_{k} \mathrm{i}, a_{k}+b_{k} q\right),
$$

where $a_{1}, b_{1}, \ldots, a_{k}, b_{k}$ are real numbers, and we have assumed $q \neq \pm \mathrm{i}$; if $q= \pm \mathrm{i}$ then we replace $\mathrm{i}$ by $\mathrm{j}$ in (3.1) .

Then for any $p \in S^{2} \backslash\{ \pm q\}$ we have $W_{k, q} \cap p W_{k, q}=0$, whilst $W_{k, q} \cap q W_{k, q}$ has dimension two. Together with [7, Proposition 3.1] this implies that the sheaf of $\left(W_{k, q}, \mathbb{H}^{k}\right)$ is the indecomposable torsion sheaf with conjugation, supported at $\pm q$, and of Chern number $2 k$.

\section{The MAIN RESUlts}

Now, we can prove the following:

Theorem 4.1 (cf. [2]). Any pair formed of a quaternionic vector space and a real vector subspace admits a decomposition, unique up to the order of factors, as a (finite) product in which each factor is given by one of the Examples 3.5, 3.6 or 3.7, or is the dual of one of the Examples 3.5 or 3.6 .

Proof. Let $\mathcal{U}$ be the sheaf of $(U, E)$. By the dual of [6, Proposition 4.7], we have that $\mathcal{U}_{-}$is the holomorphic vector bundle of a $\mathrm{CR}$ quaternionic vector space $\left(U_{-}, E_{-}\right)$. Furthermore, from the diagram of the proof of [7, Theorem 4.8] 
(adapted to the case of sheaves with conjugations) we obtain that there exists an injective morphism $t:\left(U_{-}, E_{-}\right) \rightarrow(U, E)$ which induces an injective linear map $E_{-} / U_{-} \rightarrow E / U$; equivalently, $U_{-}=E_{-} \cap t^{-1}(U)$. Therefore $t$ admits a cokernel $\left(U_{+}, E_{+}\right)$whose sheaf is, obviously, $\mathcal{U}_{+}$.

Thus, we may assume $\mathcal{U}=\mathcal{U}_{+}$and, consequently, we have an exact sequence

$$
0 \longrightarrow E^{0,1} \longrightarrow Z \times(E / U)^{\mathbb{C}} \longrightarrow \mathcal{U} \longrightarrow 0 \text {. }
$$

Then the cohomology exact sequence of (4.1) gives a canonical isomorphism (which intertwines the conjugations) $(E / U)^{\mathbb{C}}=H^{0}(Z, \mathcal{U})$.

Furthermore, the morphism $(0, E) \rightarrow(U, E)$ determines a surjective sheaf morphism $\mathcal{E} \rightarrow \mathcal{U}$ whose kernel is $Z \times U^{\mathbb{C}}$ (with the corresponding morphism to $\mathcal{E}$ given by the inclusion $Z \times U^{\mathbb{C}} \rightarrow Z \times E^{\mathbb{C}}$ followed by the projection $Z \times E^{\mathbb{C}} \rightarrow \mathcal{E}$ ). Thus, we, also, have

$$
0 \longrightarrow Z \times U^{\mathbb{C}} \longrightarrow \mathcal{E} \longrightarrow \mathcal{U} \longrightarrow 0
$$

The cohomology exact sequence of (4.2), together with the isomorphisms $(E / U)^{\mathbb{C}}=H^{0}(Z, \mathcal{U})$ and $E^{\mathbb{C}}=H^{0}(Z, \mathcal{E})$, show that the inclusion $U \rightarrow E$ is determined by $\mathcal{U}$.

Now, tensorising (4.2) with the tautological line bundle over $Z\left(=\mathbb{C} P^{1}\right)$ and by using [7, Proposition 3.1] we deduce that in the Birkhoff-Grothendieck decomposition of $\mathcal{U}$ there are no trivial terms. Together with Examples [3.5, 3.6, and 3.7, this completes the proof.

Let $(U, E)$ be a pair formed of a quaternionic vector space and a real vector subspace.

Then $(U, E)$ is a torsion pair if it corresponds to a torsion sheaf; equivalently, $(U, E)$ is a product of pairs as in Example 3.7.

The pair $(U, E)$ is torsion free if its sheaf is torsion free; equivalently, it is a holomorphic vector bundle.

Corollary 4.2. (i) Let $(U, E)$ and $\left(U^{\prime}, E^{\prime}\right)$ be pairs formed of a quaternionic vector space and a real vector subspace. Suppose that either $(U, E)$ or $\left(U^{\prime}, E^{\prime}\right)$ are torsion free. Then $\left(U \times U^{\prime}, E \times E^{\prime}\right)$ doesn't depend of the particular isometry used to define $E \times E^{\prime}$.

(ii) Any pair $(U, E)$ formed of a quaternionic vector space and a real vector subspace decomposes uniquely as the product of a torsion pair and (the pairs given by) a CR quaternionic vector space and a co- $C R$ quaternionic vector space; moreover, the filtration $(0,0) \subseteq\left(U_{-}, E_{-}\right) \subseteq\left(U_{-}, E_{-}\right) \times\left(U_{t}, E_{t}\right) \subseteq(U, E)$ is canonical, where $\left(U_{t}, E_{t}\right)$ is the torsion pair and $\left(U_{-}, E_{-}\right)$is the CR quaternionic vector space. 
Proof. If $\mathcal{U}$ is a holomorphic vector bundle over $S^{2}$ and $T: S^{2} \rightarrow S^{2}$ is a holomorphic diffeomorphism then $T^{-1}(\mathcal{U})$ is isomorphic to $\mathcal{U}$ and, furthermore, the same holds for bundles, endowed with a conjugation covering the antipodal map, and their pull-backs through orientation preserving isometries. Assertion (i) follows quickly.

Assertion (ii) follows from (i) and the proof of Theorem 4.1 .

Finally, note that the 'augmented (strengthen) $\mathbb{H}$-modules' of [5] ([7]) are just pairs whose decompositions contain no terms of the form $(\mathbb{H}, \mathbb{H})((0, \mathbb{H}))$; equivalently, in the decompositions of their sheaves there are no terms of Chern number $-1(1)$.

\section{REFERENCES}

[1] D. V. Alekseevsky, S. Marchiafava, Quaternionic structures on a manifold and subordinated structures, Ann. Mat. Pura Appl., 171 (1996) 205-273.

[2] V. Dlab, C. M. Ringel, Real subspaces of a quaternion vector space, Canad. J. Math., 30 (1978) 1228-1242.

[3] R. C. Gunning, H. Rossi, Analytic functions of several complex variables, Prentice-Hall, Inc., Englewood Cliffs, N.J. 1965.

[4] S. Ianuş, S. Marchiafava, L. Ornea, R. Pantilie, Twistorial maps between quaternionic manifolds, Ann. Sc. Norm. Super. Pisa Cl. Sci. (5), IX (2010) 47-67.

[5] D. Joyce, Hypercomplex algebraic geometry, Q. J. Math., 49 (1998) 129-162.

[6] S. Marchiafava, L. Ornea, R. Pantilie, Twistor Theory for CR quaternionic manifolds and related structures, Monatsh. Math., (in press).

[7] D. Quillen, Quaternionic algebra and sheaves on the Riemann sphere, Q. J. Math., 49 (1998) 163-198.

[8] D. Widdows, Quaternionic algebra described by $\operatorname{Sp}(1)$ representations, Q. J. Math., 54 (2003) 463-481.

E-mail address: radu.pantilie@imar.ro

R. Pantilie, Institutul de Matematică "Simion Stoilow" Al Academiei Române, C.P. 1-764, 014700, BuCureşTi, RomÂniA 